\title{
A mean field game analysis of electric vehicles in the smart grid
}

\author{
Romain Couillet $^{1}$, Samir Medina Perlaza ${ }^{2}$, Hamidou Tembine ${ }^{3}$, and Mérouane Debbah ${ }^{2}$ \\ ${ }^{1}$ Centrale-Supélec-EDF Chair on System Sciences and the Energy Challenge, Gif sur Yvette, France. \\ ${ }^{2}$ Alcatel-Lucent Chair on Flexible Radios, Supélec, Gif sur Yvette, France. \\ ${ }^{3}$ Telecommunication Department, Supélec, Gif sur Yvette, France.
}

\begin{abstract}
In this article, we develop a mean field game model for the economical analysis of the integration of purely electrical vehicles (EV) or electrical hybrid oil-electricity vehicles (PHEV) in the smart grid energy market. The framework we develop allows for a consistent analysis of the evolution of the price of electricity, of the timely demand, and possibly of the energy reserves in the grid, when EV or PHEV owners buy and sell electricity from their cars, selfishly but rationally, based on collective price incentives.
\end{abstract}

\section{INTRODUCTION}

It is widely recognized [1], [2], [3] that the future intense penetration of electrical vehicles (EV) and plug-in hybrid electrical vehicles (PHEV) in the energy market will generate an important additional energy demand, and therefore a new strain to the electricity suppliers. At the same time, EV and PHEV owners are not only energy consumers as EVs and PHEVs can charge as well as discharge their battery contents from or to the electrical grid. From this perspective, these vehicles can be seen as an additional large dimensional energy buffer to flatten the peaky daily and seasonal energy demands. That is, EVs or PHEVs can connect to the smart grid and sell their energy surpluses, when needed. It is therefore an important economical and social challenge to enforce charge and discharge policies to EVs and PHEVs in an optimal manner. Here, optimality must be interpreted in the sense of maximum individual revenue obtained by the EV and PHEV owners when participating in the energy trades, as well as maximum performance of the electricity grid, e.g. low probability for blackouts, large energy reserves, etc. The price at which the energy is sold or bought depends obviously on the total demand and offer of all electrical vehicles present in the network (and also on the existing energy consumption by ancillary services in the grid). The electricity price is set by the energy market, and we therefore assume the existence of communications between EVs and the grid. Relevant important aspects of EV-to-grid communication protocols can be found in e.g. [4], [5], [6]. Since each EV action impacts the satisfaction of all other EVs, it impact the next actions of the latter in return, all of them being therefore in competition.

This competitive interaction in which each vehicle owner decides the amount of energy to be sold or bought at any time given a global price can be analyzed using tools from dynamic game theory [7]. For instance, in [8], the coexistence of a number of PHEV groups aiming to sell part of their stored energy to the smart grid are studied using non-cooperative game theory. The authors propose in particular an algorithm based on best response dynamics to allow PHEV groups to reach a Nash equilibrium point [9]. Nonetheless, in practical scenarios, the number of vehicles is extremely large and, thus, elements from classical game theory are difficult to handle and in general do not bring enough insight about the global behavior of the market.

To overcome this problem, in this paper, we study the energy trade when the number of vehicles tends to infinity and all vehicles can be considered identical. Within this framework, we model the game as a mean field game [10], [11]. As opposed to $N$-player games where the objective is to follow the evolution of the state $X_{t}^{(i)}$ of every individual player $i \in\{1, \ldots, N\}$ at every time instant $t$ of the game, the object of mean field games is rather to obtain the optimal distribution $m^{\star}(t, X)$ of the players being in state $X$ at time $t$ (all players being in the same state at the same time behave similarly). As a main consequence, it is possible in these games to follow the state trajectory of all players at once and to capture the behavior of the players depending only on (i) their initial state $X_{0}$ and (ii) the joint distribution $m^{\star}(0, \cdot)$, at time $t=0$. The notion of Nash equilibrium in the context of mean field games is extended to the notion of mean field equilibrium [10], [11]. A mean field equilibrium is now a fixed point solution to a coupled system of stochastic partial differential equations which includes a (backward) Hamilton-Jacobi-Bellman (HJB) equation and a (forward) Fokker-Planck-Kolmogorov (FPK) equation. The HJB equation determines the optimal controls $\alpha_{t}=\alpha_{t}\left(X_{t}\right)$, or actions, of the players (the EV owners) in state $X_{t}$ at time $t$, given any density measure $m$, while the FPK equation determines the density $m$ for a given control function $\alpha_{t}$.

In [12], a mean field game approach to the study of oil production in unlimited time is developed where the selfish players are oil producers and the mean field variable is the oil selling price. In this article, we develop a similar framework to [12] but on a finite time horizon, applied to both EVs and PHEVs, with vehicle owners as the selfish players and electricity price as the mean field variable of interest. The mean-field property relates presently to the fact that every player's action is driven not by the individual actions of each other player but by the collective (or mean) behavior of all players. Indeed, we will assume that the price for electricity is driven both by the smart grid which may enforce policies 
at specific time instants but more importantly by the total amount of energy bought at time $t$ by the players. The state evolution under study will be here the evolution of the quantity $X_{t}$ of electricity in the battery of the EV owners or the joint quantity $Z_{t}=\left[X_{t} Y_{t}\right]^{\top}$ of electricity and oil in the battery and tank of PHEV owners. This state is controlled by the quantity $\alpha_{t}\left(X_{t}\right)$ of electricity, or $\left(\alpha_{t}^{X}\left(Z_{t}\right), \alpha_{t}^{Y}\left(Z_{t}\right)\right)$ of electricity and oil, bought or sold by players in state $X_{t}$, or $Z_{t}$, at time $t$.

The reminder of this article unfolds as follows. In Section II, we introduce the mean field game model for the electrical vehicle problem under consideration. In Section III, we develop the fundamental equations describing a mean field equilibrium of the systems under study. In Section IV, we run numerical simulations and derive conclusions in both study scenarios. Finally, in Section $\mathrm{V}$, we conclude this work.

Notations: The notations $\partial_{x} F(X, Y), \quad \partial_{x} F(X, Y)$, $\partial_{x x}^{2} F(X, Y)$ and $\partial_{y y}^{2} F(X, Y)$ stand for the first and second partial derivatives of the function $F$ along the variables $X$ and $Y$. The notation $d X$ for $X$ a random process is the differential notation following Itô's definition. The notation $[x]^{+}$stands for $\max (x, 0)$. The function $\delta_{A}$ is the indicator function on the set $A$.

\section{MODEL}

In this section, we develop the two models of EVs and PHEVs under study.

\section{A. Electrical vehicles}

Consider the game to be played by $N$ electrical vehicle owners who decide at all time on the optimal battery level given a utility function encompassing the economical cost or gain of filling or emptying the battery, the (psychological) cost of operating on the battery at certain time instants of the day, and the disutility for the user to have a nearly empty battery.

For player $i \in\{1, \ldots, N\}$, we denote $X_{t}^{(i)} \in[0,1]$ its state variable, corresponding to its battery level at time $t \in[0, T]$. We assume that player $i$ modifies $X_{t}^{(i)}$ by a quantity $\alpha_{t}^{(i)} d t$, at time $t$. We also assume that player $i$ consumes a quantity $g_{t}^{(i)} d t$ of energy at time $t$. We therefore have the state evolution for each player given by the equation

$$
\frac{d}{d t} X_{t}^{(i)}=\alpha_{t}^{(i)}-g_{t}^{(i)}
$$

where we implicitly assumed the differentiability of the state variable $X_{t}^{(i)}$. This supposes in particular that electricity will be bought or sold constantly, instead of by bursts. This is a rather unrealistic assumption which will be made more realistic when we consider the mean field limit.

The objective for player $i$ is to minimize the cost for an initial condition $\left\{X_{0}^{(j)}\right\}_{j}$ for all players over the time window $[0, T]$, given by

$v^{(i)}\left(0,\left\{X_{0}^{(j)}\right\}_{j} ;\left\{\alpha_{t}^{(i)}\right\}_{t},\left\{\alpha_{t}^{(-i)}\right\}_{t}\right)=$
$\int_{0}^{T}\left[\alpha_{t}^{(i)} p_{t}\left(\left\{\alpha_{t}^{(j)}\right\}_{j}\right)+h_{i}\left(t, \alpha_{t}^{(i)}\right)+f_{i}\left(t, X_{t}^{(i)}\right)\right] d t+k_{i}\left(X_{T}^{(i)}\right)$ where $\alpha_{t}^{(-i)}=\left[\alpha_{t}^{(1)}, \ldots, \alpha_{t}^{(i-1)}, \alpha_{t}^{(i+1)}, \ldots, \alpha_{t}^{(N)}\right]^{\top}$ are the controls of all players but $i$ at time $t$, the term $p_{t}\left(\left\{\alpha_{t}^{(j)}\right\}_{j}\right)$ is the price of electricity (identical for all players) given the instantaneous energy demand $\sum_{j} \alpha_{t}^{(j)}$ (we assume that both buying and selling prices are equal), $h_{i}(t, \alpha)$ is the psychological cost for player $i$ to perform a change $\alpha d t$ in the battery level at time $t$ (some time periods are more appropriate than others to purchase or sell energy depending on the player's routine), $f_{i}(t, X)$ is the cost for player $i$ to be in state $X$ at time $t$ (typically, one does not wish to have a low battery level in certain periods of time), and $k_{i}(X)$ is the (terminal) cost of being at state $X$ at time $T$ (this avoids the undesirable effect that all energy is sold in the last instants of $[0, T]$ ). Details on specific choices for these functions are provided later.

In game theory, this formulation is known as an $N$-player continuous-time differential game of pre-specified fixed duration $T$ [7]. The notion of equilibrium in this game corresponds to the state of simultaneous satisfaction of all players in the sense of Nash [9], [13]. That is, the family of controls $\left\{\alpha_{t}^{(i)}\right\}_{t, i}$ engender a Nash equilibrium if

$$
\begin{aligned}
& v^{(i)}\left(0,\left\{X_{0}^{(j)}\right\}_{j} ;\left\{\alpha_{t}^{(i)}\right\}_{t},\left\{\alpha_{t}^{(-i)}\right\}_{t}\right) \\
\leq & v^{(i)}\left(0,\left\{X_{0}^{(j)}\right\}_{j} ;\left\{\alpha_{t}^{(i)}\right\}_{t},\left\{\alpha_{t}^{(-i)}\right\}_{t}\right)
\end{aligned}
$$

for each $i$, with $\left\{\alpha_{t}^{\prime(i)}\right\}_{t}$ any admissible family of controls of player $i$ over $t \in[0, T]$.

The interest of Nash equilibria in the EV interaction lies in the fact that, at such state, all the EVs are using a control policy which is optimal with respect to the control policy of all the other EVs. Otherwise stated, if the system is at equilibrium, the unilateral deviation of any player would lead it to a higher cost and, thus, none of the players has a particular interest on unilaterally changing its control functions. However, analyzing the Nash equilibrium of a game where $N$ is a large number is a very complex problem. In fact, even if a Nash equilibrium exits, it would lead to solutions that are inherently difficult to exploit. In particular, it is clear that, under this formulation, any change in the battery level of a given player impacts all other players which must react as a consequence. Instead, we will consider a simplification of this framework by assuming a fluid limit as $N$ grows large. The foundations of this approach were established in [14]; see also [15] for recent results. Within this framework, instead of considering the vector $\left(X_{t}^{(1)}, \ldots, X_{t}^{(N)}\right)$ of state spaces at time $t$, we consider the variable $X_{t}$ with density measure $m\left(t, X_{t}\right)$ at time $t$, defined as

$$
m(t, X)=\lim _{N \rightarrow \infty} \frac{1}{N} \sum_{i=1}^{N} \delta_{\left\{X_{t}^{(i)} \leq X\right\}}(X)
$$

assuming the existence of such a fluid limit. The control variable is now a variable $\alpha_{t}$ with density. Since it would be inappropriate to assume that all individual players consume exactly the same density of electricity per time instant, we consider instead that the energy consumption is a random variable with mean $g_{t} d t$ and with variance $\sigma_{t}^{2}$ at time $t$; this is modelled through the variable $g_{t}\left[d t+\sigma_{t} d W_{t}\right]$, where $W_{t}$ is a Brownian motion and the differential notation $d W_{t}$ must be interpreted in the sense of Itô [16]. All other cost functions are 
supposed identical for all players, so we substitute in particular the functions $h, k$ and $f$ to $h_{i}, k_{i}$ and $f_{i}$, respectively, and denote $p_{t}(m(t, \cdot))$ the price of electricity given the density $m(t, \cdot)$ at time $t$.

With these updated notations, the objective for any given user is to solve the following stochastic control problem

$$
\begin{aligned}
v\left(0, X_{0}\right) & =\inf _{\left(\alpha_{t}\right)_{t \in[0, T]}} \mathrm{E}\left[\int_{0}^{T} C\left(\alpha_{t}, X_{t}, m, t\right) d t+k\left(X_{T}\right)\right] \\
d X_{t} & =\alpha_{t} d t-g_{t}\left[d t+\sigma_{t} d W_{t}\right]+d N_{t}
\end{aligned}
$$

where $d N_{t}$ is a reflective variable to ensure that $X_{t}$ remains in $[0,1]$.

Regarding the cost function, $C$ is given by

$$
C\left(\alpha_{t}, X_{t}, m, t\right)=\alpha_{t} p_{t}(m(t, \cdot))+h\left(t, \alpha_{t}\right)+f\left(t, X_{t}\right) .
$$

It would stand then to reason to consider that $p_{t}(m)$ writes as a function of the total instantaneous demand $\int \alpha_{t}(X) m(t, X) d X$. However, for computational ease, we will instead consider that prices are fixed not by the total consumption $\int \alpha_{t}(X) m(t, X) d X$ but by the total anticipated consumption $g_{t}+\frac{d}{d t}\left[\int X m(t, X) d X\right]$, where both quantities only differ by an additional Brownian motion term when $\sigma_{t}>0$. That is, we assume that the energy providers do not have the information on the instantaneous demand at time $t$ but are able to track the density $m(t, \cdot)$ at all time. This is a reasonable assumption as this only requires for the market to obtain the status information feedback from a not-necessarily large sample of electrical vehicles at different (sampled) time instants.

We therefore define $p_{t}$ as

$$
p_{t}(m)=D(t, \cdot)^{-1}\left(g_{t}+\frac{d}{d t} \int X m(t, X) d X\right)
$$

where $D(t, p)$ is the total energy demand function at time $t$ for a given price $p$. This function is central to create incentive behavior among players. For instance, at peak demand periods for electricity services other than EVs, $p_{t}$ may be increased for negative $\mathrm{EV}$ electricity demand and decreased for positive EV electricity demand so to enforce car owners to sell rather than buy electricity.

This fluid limit formulation now defines a mean field game, as introduced in [10], [11]. Our specific problem setting is as a matter of fact very close to the work [12]. In this framework, the solutions to the control problem (1) can be seen as generalizations of Nash equilibria [9] in the equivalent $N$-person game. It is in general difficult to prove that the mean field solutions are well-defined limits of the $N$-person game, see e.g. the discussions in [17]. Similarly, it is in general difficult to prove the existence and uniqueness of solutions to (1) and, if so, to derive a numerical method that is provably converging to the solution as the discretization step tends to zero, see e.g. [18]. We will not try to prove any of these aspects here, our main target being rather to informally explore the potentials of the mean field game setting to the economical problem of optimal policies for EV and PHEV penetrations in the smart grid. Similar to [12], only the numerical results will convey a justification of the correct behavior of our method.
In the next section, we improve the EV framework by turning the purely electrical vehicles into PHEVs, introducing therefore the possibility for players to select between two alternative sources of energy.

\section{B. Hybrid vehicles}

We now consider that players choose to buy, sell and consume either electricity with the elastic price defined as in Section II-A or a fix-priced alternative energy source, oil. We consider immediately the mean-field game limit. In this scenario, the state function becomes a two-dimensional vector $Z_{t}=\left[\begin{array}{ll}X_{t} & Y_{t}\end{array}\right]^{\top}$, where $Y_{t} \in[0,1]$ is the content of the oil tank. We now replace the control $\alpha_{t}$ by a vector $\gamma_{t}=\left[\begin{array}{ll}\alpha_{t}^{X} & \alpha_{t}^{Y}\end{array}\right]^{\top}$, where $\alpha_{t}^{X} \in \mathbb{R}$ is the content of electricity bought or sold by the vehicle owner at time $t$ and $\alpha_{t}^{Y} \geq 0$ is the oil content bought by the owner at time $t$ (oil cannot be sold once bought). In addition, we denote $\beta\left(t, Z_{t}\right) \in[0,1]$ the relative quantity of electricity being consumed by the PHEVs at time $t$. That is, we assume that all vehicles have a common policy of energy consumption in the considered time window. Typically, taking $\beta\left(t, Z_{t}\right)=X_{t} /\left(X_{t}+Y_{t}\right)$ translates a policy where energy is consumed indistinctly of the energy source. Note that, depending on the typical distances covered by PHEV owners at time $t$ (e.g. weekdays against weekends), $\beta\left(t, Z_{t}\right)$ may explicitly depend on $t$. Alternatively, we may have considered $\beta\left(t, Z_{t}\right)$ an additional control variable which can be set optimally by the car owner depending on the status of the energy market. For simplicity of analysis and because this involves a somewhat unrealistic constant calculus from the PHEV owners, we do not consider this scenario here. We now call $m$ the density for the variable $Z_{t}$.

The price for electricity is given by the function $p_{t}^{X}(m)$ updated as follows:

$$
\begin{aligned}
& p_{t}^{X}(m)= \\
& D(t, \cdot)^{-1}\left(g_{t} \int_{Z} \beta(t, Z) m(t, Z) d Z+\frac{d}{d t} \int_{X} X m(t, Z) d X\right) .
\end{aligned}
$$

The price for oil is given by $p^{Y}=p_{t}^{Y}$, supposed constant throughout $[0, T]$, which is a satisfying assumption on a daily or even weekly basis. The optimal control problem now formulates as:

$$
\begin{aligned}
v\left(0, Z_{0}\right) & =\inf _{\left(\gamma_{t}\right)} \mathrm{E}\left[\int_{0}^{T} C\left(\gamma_{t}, Z_{t}, m, t\right) d t+k\left(Z_{T}\right)\right] \\
d Z_{t} & =\left[\begin{array}{c}
\alpha_{t}^{X} \\
\alpha_{t}^{Y}
\end{array}\right] d t-\left[\begin{array}{c}
\beta\left(t, Z_{t}\right) \\
1-\beta\left(t, Z_{t}\right)
\end{array}\right] g_{t}\left[d t+\sigma_{t} d W_{t}\right]+d N_{t}
\end{aligned}
$$

where $v\left(0, Z_{0}\right)=v\left(0, Z_{0} ;\left\{\gamma_{t}\right\}_{t}\right)$ assumes the dependence on $\left\{\gamma_{t}\right\}_{t}$ and where

$$
C\left(\gamma_{t}, Z_{t}, m, t\right)=\alpha_{t}^{X} p_{t}^{X}(m)+\alpha_{t}^{Y} p^{Y}+h\left(t, \gamma_{t}\right)+f\left(t, Z_{t}\right)
$$

with similar notations as previously.

Now that both mean field game formulations have been laid out, we concentrate on solving these optimal control problems in the next section. 


\section{MEAN FIELD EQUILIBRIUM}

In this section, we analyze the mean field game described in the previous section. In this context, the notion of Nash equilibrium introduced previously is extended to the notion of mean field equilibrium [10], [11].

As already mentioned, the mean field equilibrium is obtained as a solution to a coupled system of stochastic partial differential equations constituted by a backward HJB equation and a forward FPK equation. In this section, we detail these fundamental equations in the EV and PHEV frameworks of Section II-A and Section II-B, respectively.

\section{A. Electrical vehicles}

Consider the optimization problem of Section II-A, and define the following value function,

$$
v\left(u, X_{u}\right)=\inf _{\left(\alpha_{t}\right)_{t \in[u, T]}} \mathrm{E}\left[\int_{u}^{T} C\left(\alpha_{t}, X_{t}, m, t\right) d t+k\left(X_{T}\right)\right] .
$$

Then, the measure $m^{\star}\left(t, X_{t}\right)$ and the control functions $\alpha_{t}^{\star}$ at the mean field equilibrium satisfy the following system of coupled differential equations given the initial distribution $m(0, \cdot)$. First, an HJB equation determines the optimal trajectory $v^{\star}\left(t, X_{t}\right)$ in the control problem (1). That is,

$$
\begin{aligned}
0 & =\partial_{t} v(t, X)+\inf _{\alpha}\left\{\alpha \partial_{x} v(t, X)+C\left(\alpha, X, m^{\star}(t, X), t\right)\right\} \\
& -g_{t} \partial_{x} v(t, X)+\frac{1}{2} g_{t}^{2} \sigma_{t}^{2} \partial_{x x}^{2} v(t, X) .
\end{aligned}
$$

Then, an FPK equation determines the measure $m^{\star}\left(t, X_{t}\right)$ of the game state. Precisely, this is

$$
\partial_{t} m(t, X)=-\partial_{x}\left[\left(\alpha_{t}^{\star}-g_{t}\right) m(t, X)\right]+\frac{1}{2} g_{t}^{2} \sigma_{t}^{2} \partial_{x x}^{2} m(t, X)
$$

where $\alpha_{t}^{\star}$ is the argument of the infimum in (2). Details of how these equations are obtained can be found in e.g. [19].

We assume here that the cost $h(t, \alpha)$ for control is quadratic and reads

$$
h(t, \alpha)=\frac{1}{2} h_{t} \alpha^{2}
$$

with $h_{t}>0$ representing the unwillingness of the car owner to buy or sell energy at time $t$. This choice is seemingly non-natural as it implies that users are more willing to buy or sell small quantities rather than large quantities of energy. Nonetheless, under the mean field game formulation, this has to be understood as the fact that, on average, only a limited population of users at time $t$ is willing (or able) to buy energy. For instance, during nighttime, many EVs are plugged to an electrical source so that $h_{t}$ is smaller at night (more users can ask for energy). As such, intuitively, making the (psychological) cost of buying or selling energy larger for larger amounts of energy forces only part of the population to buy or sell. As for the particular choice of a quadratic cost rather than any other cost function, it is convenient for calculus mostly and could be easily replaced by more appropriate functions.
Under these conditions, from Pontryagin's minimum principle [19], we immediately have an expression of the optimal control $\alpha_{t}^{\star}$ as the minimizer of

$$
\inf _{\alpha}\left\{\alpha \partial_{x} v\left(t, X_{t}\right)+C\left(\alpha, X, m^{\star}, t\right)\right\} .
$$

Precisely, we have

$$
\alpha_{t}^{\star}=-\frac{1}{h_{t}}\left[\partial_{x} v\left(t, X_{t}\right)+p_{t}\left(m^{\star}\right)\right]
$$

possibly submitted to some boundary conditions to ensure that $X_{t} \in[0,1]$ at all times. In the remainder of the article, we will assume this condition always met, so that at no time we will consider EV owners with completely full or completely empty batteries.

The HJB equation now becomes

$$
\begin{aligned}
0 & =\partial_{t} v(t, X)-\left(\frac{1}{h_{t}}\left[\partial_{x} v(t, X)+p_{t}\left(m^{\star}\right)\right]+g_{t}\right) \partial_{x} v(t, X) \\
& -\frac{p_{t}\left(m^{\star}\right)}{h_{t}}\left[\partial_{x} v(t, X)+p_{t}\left(m^{\star}\right)\right]+f(t, X) \\
& +\frac{1}{2 h_{t}}\left[\partial_{x} v(t, X)+p_{t}\left(m^{\star}\right)\right]^{2}+\frac{1}{2} \sigma_{t}^{2} g_{t}^{2} \partial_{x x}^{2} v(t, X)
\end{aligned}
$$

which can be simplified as

$$
\begin{aligned}
\partial_{t} v(t, X) & =\frac{1}{2 h_{t}}\left(\partial_{x} v(t, X)+p_{t}\left(m^{\star}\right)\right)^{2}+g_{t} \partial_{x} v(t, X) \\
& -f(t, X)-\frac{1}{2} \sigma_{t}^{2} g_{t}^{2} \partial_{x x}^{2} v(t, X)
\end{aligned}
$$

and the FPK equation is

$$
\begin{aligned}
& \partial_{t} m(t, X)= \\
& \left(\frac{1}{h_{t}}\left[\partial_{x} v^{\star}(t, X)+p_{t}(m(t, X))\right]+g_{t}\right) \partial_{x} m(t, X) \\
& +\frac{1}{h_{t}} \partial_{x x}^{2} v^{\star}(t, X) m(t, X)+\frac{1}{2} g_{t}^{2} \sigma_{t}^{2} \partial_{x x}^{2} m(t, X) .
\end{aligned}
$$

This defines the two fundamental differential equations to be solved for the optimal EV policy. We now turn to the resolution of the PHEV problem.

\section{B. Hybrid electric-oil vehicles}

Under the notations of Section II-B, and similar to the previous section, we consider the value function

$$
v\left(u, Z_{u}\right)=\inf _{\left(\gamma_{t}\right)_{t \in[u, T]}} \mathrm{E}\left[\int_{u}^{T} C\left(\gamma_{t}, Z_{t}, m, t\right) d t+k\left(Z_{T}\right)\right]
$$

where we remind that $\left.Z_{t}=\left[\begin{array}{ll}X_{t} & Y_{t}\end{array}\right]^{\top}\right]$ is a two-dimensional vector, with a given initial distribution $m(0, \cdot)$.

As in the EV formulation, we consider the cost function $h$ as quadratic, as follows

$$
h\left(t, \gamma_{t}\right)=\frac{1}{2} h_{t}^{X}\left(\alpha_{t}^{X}\right)^{2}+\frac{1}{2} h_{t}^{Y}\left(\alpha_{t}^{Y}\right)^{2} .
$$

The HJB equation is here given by

$$
\begin{aligned}
& -\partial_{t} v \\
& =\inf _{(\gamma)}\left\{p_{t}^{X} \alpha^{X}+p^{Y} \alpha^{Y}+h(t, \gamma)+\left(\alpha^{X}-g_{t} \beta\right) \partial_{x} v\right. \\
& \left.+\left(\alpha^{Y}+g_{t}(\beta-1)\right) \partial_{y} v\right\}+f\left(t, Z_{t}\right) \\
& +\frac{1}{2} \sigma_{t}^{2} g_{t}^{2}\left[\beta^{2} \partial_{x x}^{2} v+2 \beta(1-\beta) \partial_{x, y}^{2} v+(1-\beta)^{2} \partial_{y y}^{2} v\right]
\end{aligned}
$$


with notation $\gamma=\left[\alpha^{X} \alpha^{Y}\right]$ and $\beta=\beta\left(t, Z_{t}\right)$.

Due to the parameter decoupling, the optimal $\left(\alpha_{t}^{X}\right)^{\star}$ and $\left(\alpha_{t}^{Y}\right)^{\star}$ are easily found as

$$
\begin{aligned}
& \left(\alpha_{t}^{X}\right)^{\star}=-\frac{1}{h_{t}^{X}}\left(p_{t}^{X}\left(m^{\star}\right)+\partial_{x} v\right) \\
& \left(\alpha_{t}^{Y}\right)^{\star}=-\frac{1}{h_{t}^{Y}}\left(p^{Y}+\partial_{y} v\right)
\end{aligned}
$$

with possibly additional constraints to ensure $\left(\alpha_{t}^{Y}\right)^{\star} \geq 0$ and $Z_{t} \in[0,1]^{2}$. We will assume all these conditions met for simplicity.

The FPK equation is in turn given by

$$
\begin{aligned}
& \partial_{t} m= \\
& -\partial_{x}\left[\left(\alpha_{t}^{X \star}-\beta g_{t}\right) m\right]-\partial_{y}\left[\left(\alpha_{t}^{Y \star}+(\beta-1) g_{t}\right) m\right] \\
& +\frac{1}{2} g_{t}^{2} \sigma_{t}^{2}\left[\beta^{2} \partial_{x x}^{2} m+(1-\beta)^{2} \partial_{y y}^{2} m+2 \beta(1-\beta) \partial_{x y}^{2} m\right]
\end{aligned}
$$

where we remind that $\beta=\beta\left(t, Z_{t}\right)$ depends explicitly on $Z_{t}$.

Assuming $\sigma_{t}=0$ to obtain compact forms, we then have, after substitution, the HJB equation

$$
\begin{aligned}
\partial_{t} v & =\frac{1}{2 h_{t}^{X}}\left(\partial_{x} v+p_{t}^{X}\left(m^{\star}\right)\right)^{2}+\frac{1}{2 h_{t}^{Y}}\left(\partial_{y} v+p_{t}^{Y}\right)^{2} \\
& +g_{t} \beta \partial_{x} v+g_{t}(1-\beta) \partial_{y} v-f
\end{aligned}
$$

where $m^{\star}$ is solution to the FPK equation

$$
\begin{aligned}
\partial_{t} m & =\left[\frac{1}{h_{t}^{X}}\left(\partial_{x x}^{2} v^{\star}\right)+\frac{1}{h_{t}^{Y}}\left(\partial_{y y}^{2} v^{\star}\right)+g_{t}\left[\partial_{x} \beta-\partial_{y} \beta\right]\right] m \\
& +\left[\frac{1}{h_{t}^{X}}\left(p_{t}^{X}(m)+\partial_{x} v^{\star}\right)+\beta g_{t}\right] \partial_{x} m \\
& +\left[\frac{1}{h_{t}^{Y}}\left(p^{Y}+\partial_{y} v^{\star}\right)+(1-\beta) g_{t}\right] \partial_{y} m .
\end{aligned}
$$

with $v^{\star}$ the solution to the HJB equation.

For $\beta\left(t, Z_{t}\right)=\frac{X_{t}}{X_{t}+Y_{t}}$, we have in particular

$$
g_{t}\left[\partial_{x} \beta-\partial_{y} \beta\right] m=\frac{1}{X_{t}+Y_{t}} g_{t} m .
$$

In all generality, we cannot go further with the resolution of these equations which need to be solved numerically. This is the target of the next section in which simulation results are provided.

\section{Simulations}

In this section, we provide simulation results for the electrical vehicle schemes developed in Section II-A and Section II-B.

\section{A. EV analysis}

We first consider the scenario of Section II-A. We assume a realistic 24-hour scenario where players have an average consumption rate and a possibility to buy or sell that depend on specific periods of the day ( $t=0$ stands for midnight and $t=1$ for midnight a day after). However, for the sake of simplified interpretation, we consider only a rough approximation of the system parameters. Note in particular that all parameters chosen below are designed in the interest of readability of the results and do not rely on explicit models taken from the literature.

Specifically, the demand function $D(t, p)$ is an affine function of the price. We take here $p=(D(t, p))^{+}+0.5$, which ensures a positive selling price. The major trend is satisfying as it assumes that the more electricity is drained from the smart grid, the more expensive additional withdraws of energy. The threshold $p_{t} \geq 0.5$ provides a price for the electricity sold. Also note that $D$ does not depend explicitly on $t$ and, therefore, we assume here that the reserves in the electricity grid do not fluctuate sufficiently during the day to impact the price of the electricity dedicated to electrical vehicles. We may typically have chosen to change the threshold 0.5 so to encourage selling electricity at peak hours. We take the mean consumption rate $g_{t}$ in such a way that $g_{t}>0$ at any time and with two consumption peaks in the morning and in the evening (to model e.g. EV owners leaving to work and returning). This is depicted precisely in Figure 1 . The variance $\sigma_{t}^{2}$ on the consumption is taken equal to 0.01 at all time, ensuring a standard deviation of the order of $10 \%$. We then consider $h_{t}$ to be maximal at night (since most EVs are then plugged) and minimal in the middle of the day (assuming that most EVs cannot be plugged at workplaces). This is given precisely in Figure 2. The cost $f$ is given by $f(t, X)=10(1-X)^{2}$ in order to constrain the players to fill their batteries. Finally, the boundary and initial constraints for the resolution of the system of HJB and FPK equations enforce the following policies:

- $m(0, \cdot)$ is a triangle distribution centered at 0.5 and with support $[0.3,0.7]$,

- $v(T, X)=2(1-X)^{2}$ is a cost on being far from $X=1$,

- $\partial_{x} m(0, \cdot)=\partial_{x} m(1, \cdot)=\partial_{x} v(0, \cdot)=\partial_{x} v(1, \cdot)=0$ in order to force the energy content to lie in $[0,1]$.

To solve the system of equations in $(m, v)$, we proceed by solving sequentially the HJB and FPK equations using a simple fixed-point algorithm until convergence. We do not ensure here that this algorithm does converge, neither do we ensure that the solution obtained is the solution sought for. Using a finite difference method on a sampling of 192 points on both time and battery level axes, the above scheme leads to the density evolution $m^{\star}$ depicted in Figure 3. A few observations can be already made from this figure. From $t=0$ to $t=0.1$, the tendency is for the EVs to charge large amounts of electricity, which is due to the fact that players are more willing to charge in nighttime than in daytime. Then, due to both the increase in consumption and the decreased willingness to buy electricity (or the impossibility to do so for some players) around $t=0.3$, the average quantity diminishes. The low consumption at $t=0.5$, in spite of the low willingness to buy electricity, then generates a slight increase in the mean. At $t=0.7$, the sharp electricity consumption then generates a large loss. After $t=0.8$, electricity is bought again so to satisfy the final conditions. It is interesting to note that, due to the small variance $\sigma_{t}^{2}$ that was chosen, the overall tendency is for $m^{\star}$ to concentrate into a single mass. This is a usual phenomenon which determines the steady state if time were to continue with constant values for all time-dependent system parameters. 


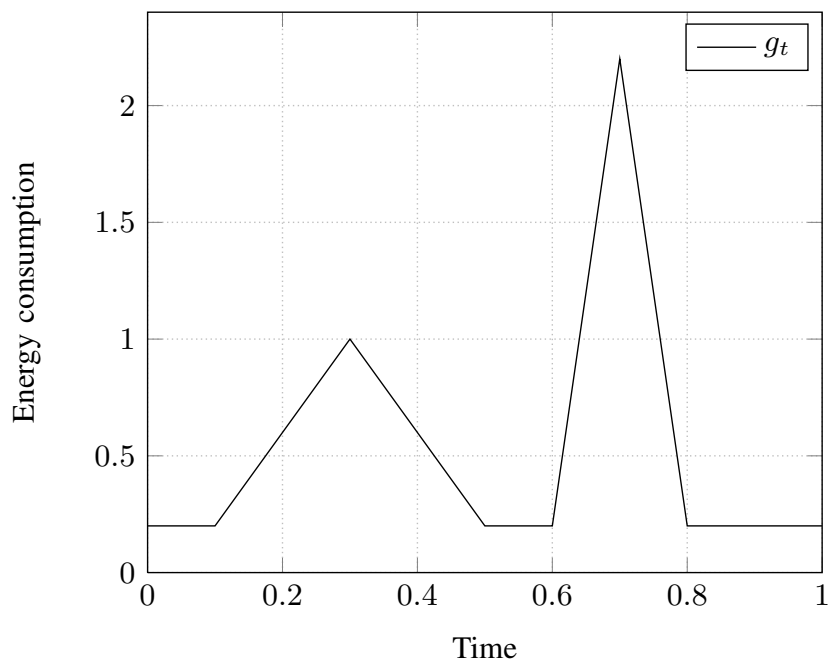

Fig. 1. Mean energy consumption $g_{t}$ of EVs as a function of time.

From the expression of $m^{\star}, v^{\star}$, and the equations derived in Section III, it is now possible to obtain much information about the system. In particular, it is interesting to follow the electricity demand $D\left(t, p_{t}\left(m^{\star}\right)\right)$ and the price $p_{t}\left(m^{\star}\right)$ defined here as (we remind that both quantities only differ by an additional term 0.5 if both are positive)

$$
p_{t}\left(m^{\star}\right)=0.5+\left[g_{t}+\frac{d}{d t} \int X m^{\star}(t, X) d X\right]^{+} .
$$

This is depicted in Figure 4, where we see that the overall tendency is to buy larger amounts of electricity early in time. The price is therefore decreasing with time but for a slight increase around the peak time $t=0.7$. The price curve has very sharp edges that can be interpreted as before as a consequence of the evolution of $g_{t}$ and $h_{t}$ mainly. With this elementary linear price policy, it is therefore possible to reduce the expected refills at peak hours, e.g. around $t=0.7$. Obviously, this interpretation has to be taken with extreme care. In particular, note that at $t=1$ the demand is very low, while it should coincide with the demand at time $t=0$ if we had been analyzing a weekly or monthly behavior instead of a single day behavior. Such a property should appear if the terminal cost constraint were reinforced.

\section{B. PHEV analysis}

In this second section, we wish to analyze the behavior of hybrid vehicles as described in Section II-B. Since solving three-dimensional differential equations is time-consuming, we only provide results for the time scale discretized in 12 samples and for the "spatial" scales discretized both in 16 samples. For each differential equation, the resolution is performed by iterating the resolution of the two-dimensional differential equations along time and electricity scales for each fixed oil tank level, and time and oil scales for each fixed battery level. Then the system of HJB and FPK differential equations is solved by further iterating a fixed point algorithm as in the previous section. For simplicity of interpretation, we consider here a time-independent scenario where both

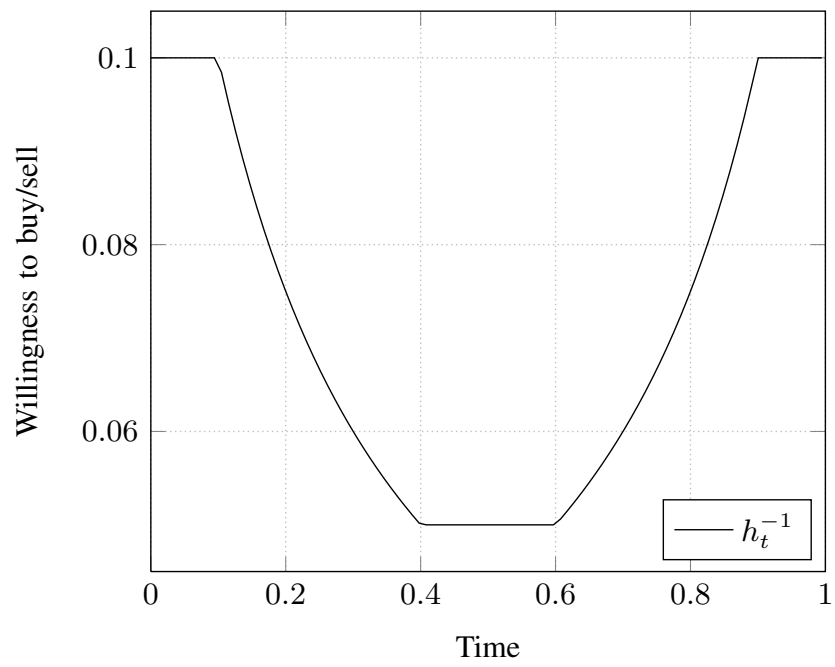

Fig. 2. Willingness $h_{t}^{-1}$ of players to operate energy transactions as a function of time.

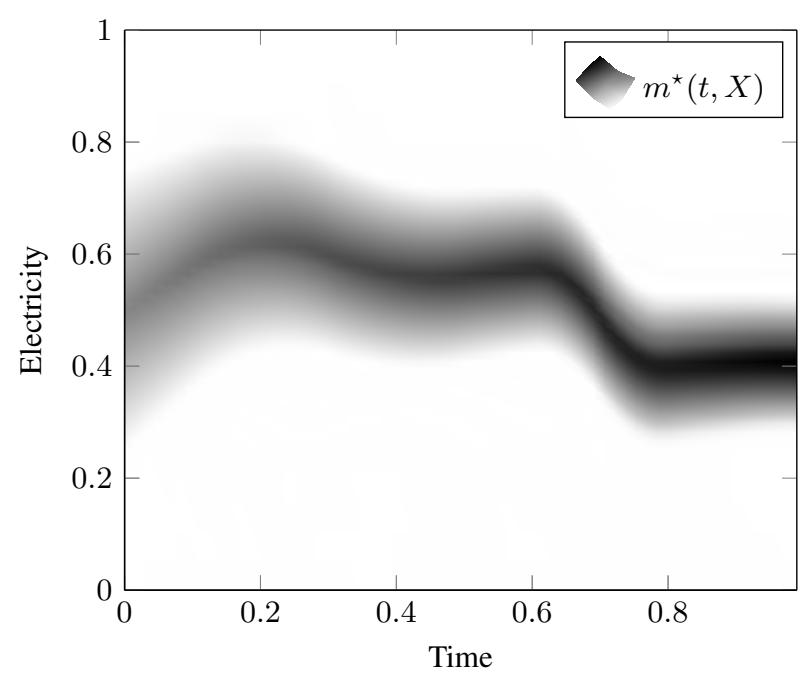

Fig. 3. Density solution $m^{\star}(t, X)$ as a function of the time $t$ and the battery level $X$.

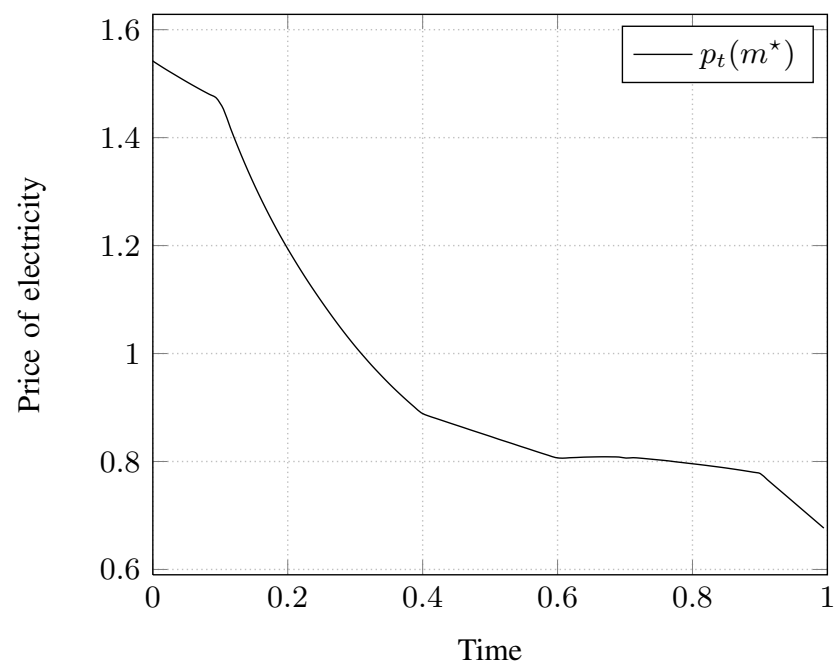

Fig. 4. Evolution of the price $p_{t}\left(m^{\star}\right)$ as a function of the time $t$. 
$g_{t}=0.2$ and $h_{t}=(125,125)^{\top}$ are constant with time. ${ }^{1}$ We take the electricity price policy to be, as above, $p^{X}=$ $\left(D\left(t, p^{X}\right)\right)^{+}+0.5$. The oil price is set to $p^{Y}=0.7$. This is a natural choice as it is expected that an approximate quantity $g_{t}=0.2$ will be asked for at any time to cover for the energy consumed, hence a price for electricity $p_{t}^{X} \simeq 0.7$. We impose a constraint $f(t, Z)=20(2-X-Y)^{2}$, where $Z=[X Y]^{\top}$. The relative consumption $\beta$ of oil and electricity is proportional to the total quantity of energy, that is $\beta(t, Z)=X /(X+Y)$ and therefore $1-\beta(t, Z)=Y /(X+Y)$. We take $\sigma_{t}=0$ for simplicity. The boundary constraints are identical to those in the previous section. As for the terminal constraint on $v$, it imposes that $v(T, Z)=10(2-(X+Y))^{2}$.

We consider the scenario where $m(0, \cdot)$ is a (properly truncated and scaled) Gaussian distribution with mean $(0.4,0.6)^{\top}$ and covariance $0.02 I_{2}$, with $I_{2}$ the $2 \times 2$ identity matrix. That is, we assume that, initially, most vehicles have more oil than electricity. This is depicted in Figure 5. We then let the system evolve freely under the above set of constraints. It is natural to guess that the overall behavior is a decrease of either or both quantities of oil and electricity to zero if the prices are too high, or an increase of either or both quantities to one, if the prices are more reasonable. What is interesting to observe is the trajectory jointly followed by the players. The resulting distribution $m^{\star}(1, \cdot)$ is depicted in Figure 6 . What we observe in the aforementioned conditions is that the initial distribution has shifted towards an increase of both electricity and oil levels, with a bigger increase of the mean battery level. Another observation is that the distribution tends to stretch along the $X=Y$ diagonal in the figure, translating the fact that oil and electricity are seen almost as equivalent goods due to the loosely constraining energy cost policy.

Among the different further analyses, in Figure 7 , we consider a section of the distribution of the optimal transaction policy $\left(\alpha_{t}^{X}\right)^{\star}$ and $\left(\alpha_{t}^{Y}\right)^{\star}$ at time $t=0^{+}$, for $Y_{t}=0.5$ and $Y_{t}=0.9$ (we remind that both $\left(\alpha_{t}^{X}\right)^{\star}$ and $\left(\alpha_{t}^{Y}\right)^{\star}$ are functions of $t, X_{t}$ and $\left.Y_{t}\right)$. That is, we observe the initial behavior of players with half-filled oil tanks and almost completely filled oil tanks. It is observed that, for users with a very low level of electricity, buying electricity is an appealing choice. This can be interpreted by the fact that, as few players are in strong need for energy, it is possible to acquire a large quantity of electricity at a reasonable price. Those players with low reserves of electricity are the main beneficiaries. For users with already a reasonable level of electricity though, electricity and oil are seen as equivalent goods. As a matter of fact, our results also show that, at time $t=0^{+}$, the price of electricity equals $p_{t}^{X}=0.706 \simeq p_{t}^{Y}$. That is, the players with low electricity levels draw as much electricity as is needed to reach an equilibrium price with oil. Now, it is also observed that, for users with large quantities of oil, electricity becomes a compelling purchase in order to further increase the total quantity of energy (since $f$ imposes $X_{t}+Y_{t}$ to be close to 2 ), hence a larger incentive for buying electricity when the battery level is not large. When both battery and tank levels

\footnotetext{
${ }^{1}$ Such a large value for the entries of $h_{t}$ is motivated by faster algorithm convergence reasons, although it inhibits as a counterpart fast variations of $m$ along time.
}

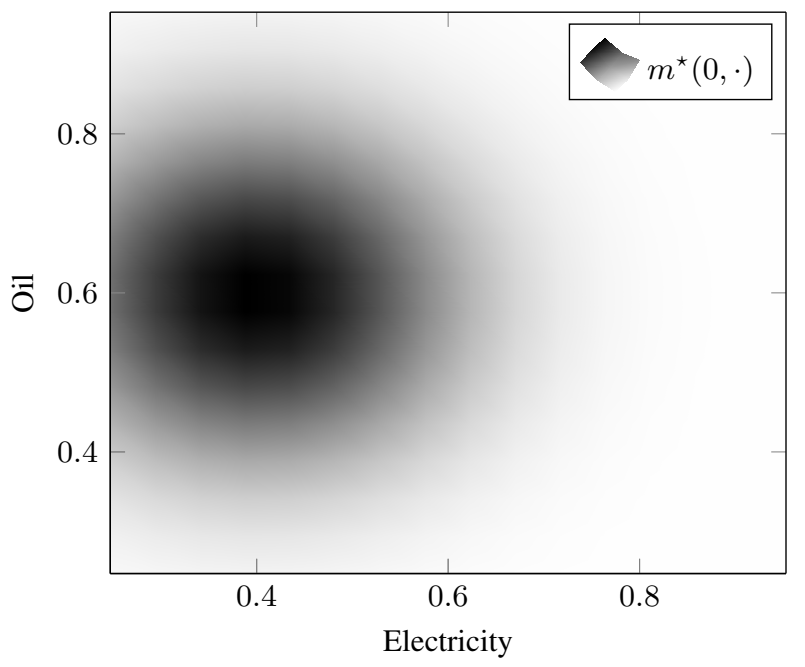

Fig. 5. Initial distribution $m(0, \cdot)$ at time $t=0$, as a function of both levels of battery and oil tank.

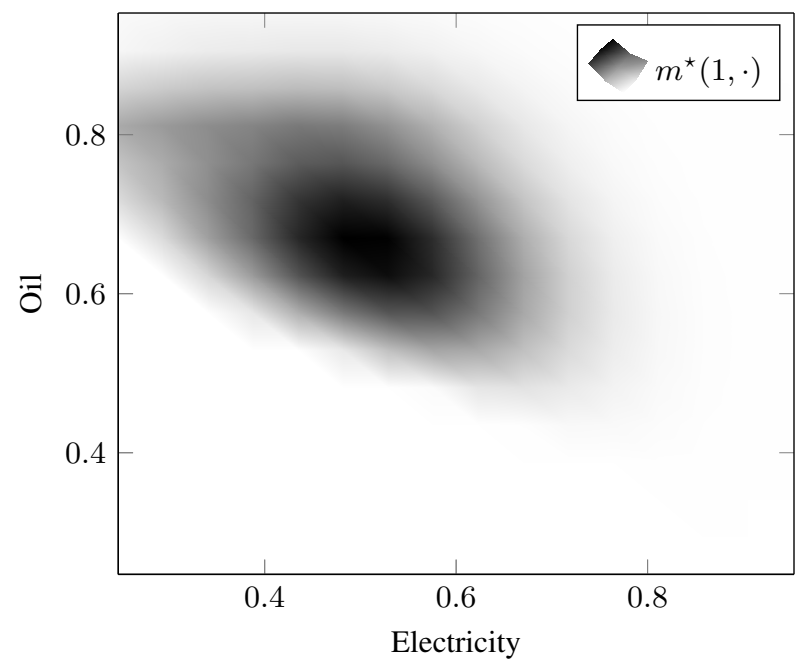

Fig. 6. Final distribution $m(1, \cdot)$ at time $t=1$, as a function of both levels of battery and oil tank.

are alike, we see that the quantity of electricity purchased is the same as the quantity of oil purchased.

Obviously, from the very generic setting of both EV and PHEV problems, many more scenarios can be carried out so to evaluate the actual impact of the EVs and PHEVs on realistic smart grid scenarios. The simulations above and their interpretations only provide a framework of understanding of fully rational vehicle owner's behavior.

\section{CONCLUSION}

In this article, we proposed a game theoretical framework to model the behavior of electrical vehicle and hybrid vehicle owners aiming at selfishly maximizing their satisfaction, under electricity pricing policy constraints. As the number of selfish players is large, players are alike, and the pricing policy depends on the action of all participants, we then turned the problem into a mean field game, for which we obtain the fundamental differential equations. We solved these equations 


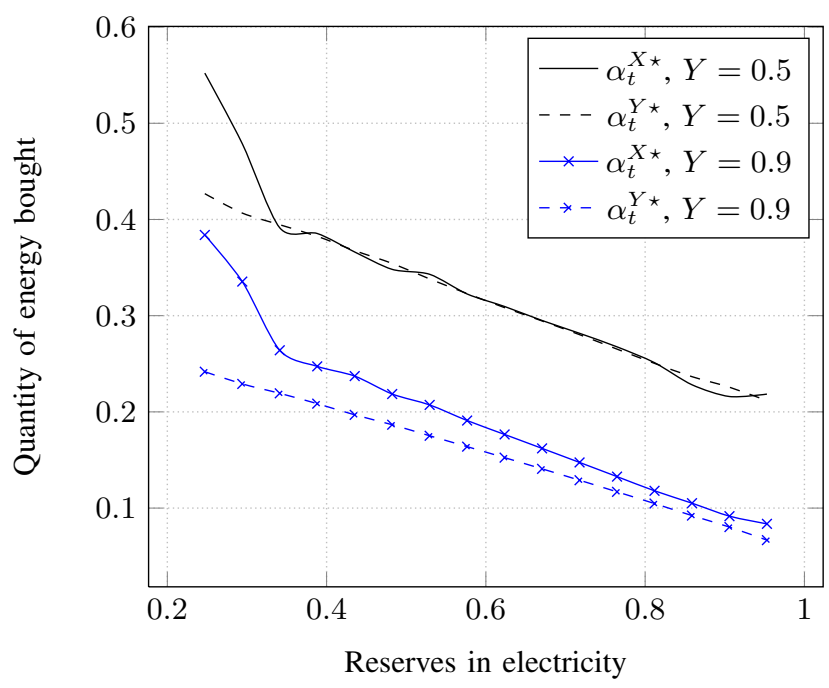

Fig. 7. Optimal transactions at time $t=0^{+}$for players with different oil and battery levels.

numerically, from which conclusions were drawn which give new insights on the way to optimize the electrical vehicle penetration in the smart grid.

\section{REFERENCES}

[1] A. Simpson, "Cost-benefit analysis of plug-in hybrid electric vehicle technology," in 22nd International Battery, Hybrid and Fuel Cell Electric Vehicle Symposium, Yokohama, Japan, Oct. 2006.

[2] T. H. Bradley and A. A. Frank, "Design, demonstrations and sustainability impact assessments for plug-in hybrid electric vehicles," Elsevier Renewable and Sustainable Energy Reviews, vol. 13, pp. 115-128, Jan. 2009.

[3] M. Kintner-Meyer, K. Schneider, and R. Pratt, "Impacts assessment of plug-in hybrid vehicles on electric utilities and regional us power grids. part i: Technical analysis," Pacific Northwest National Laboratory, 2007.

[4] E. Tara, S. Shahidinejad, S. Filizadeh, and E. Bibeau, "Battery storage sizing in a retrofitted plug-in hybrid electric vehicle," IEEE Transactions on Vehicular Technology, vol. 59, no. 6, pp. 39-50, 2010.

[5] J. Gonder, T. Markel, M. Thornton, and A. Simpson, "Using global positioning system travel data to assess real-world energy use of plugin hybrid electric vehicles," Transportation Research Record: Journal of the Transportation Research Board, vol. 2007, no. 2017, pp. 26-32, 2008.

[6] F. Pan, R. Bent, A. Berscheid, and D. Izraelevitz, "Locating PHEV exchange stations in V2g," in International Conference on Smart Grid Communications, Gaithersburg, MD, USA, Oct. 2010, Oct. 2010.

[7] T. Başar and G. J. Olsder, Dynamic Noncooperative Game Theory. Philadelphia, PA, USA: SIAM Series in Classics in Applied Mathematics.

[8] W. Saad, Z. Han, H. V. Poor, and T. Başar, "A noncooperative game for double auction-based energy trading between PHEVs and distribution grids," in The 2nd IEEE International Conference on Smart Grid Communications (SmartGridComm), Brussels, Belgium, Oct. 2011.

[9] J. F. Nash, "Equilibrium points in $n$-person games," Proceedings of the National Academy of Sciences of the United States of America, vol. 36, no. 1 , pp. $48-49,1950$.

[10] J. M. Lasry and P. L. Lions, "Jeux à champ moyen. i - le cas stationnaire," Comptes Rendus Mathématique, vol. 343, no. 9, pp. 619625, 2006

[11] — "Jeux à champ moyen. ii - horizon fini et contrôle optimal," Comptes Rendus Mathématique, vol. 343, no. 10, pp. 679-684, 2006.

[12] O. Guéant, J. M. Lasry, and P. L. Lions, "Mean field games and oil production," Preprint, 2010

[13] J. P. Aubin, "Mathematical methods of game and economic theory," Studies in Mathematics and its application, 1976.

[14] R. Aumann, "Markets with a continuum of trackers," Econometrica, vol. 32, pp. 39-50, 1964.
[15] G. Carmona, "Nash equilibria of games with a continuum of players," Reprint, 2004

[16] K. Itô, "Multiple Wiener integral," J. Math. Soc. Japan, vol. 3, no. 1, pp. 157-169, 1951

[17] O. Guéant, J. M. Lasry, and P. L. Lions, "Mean field games and applications," Paris-Princeton Lectures on Mathematical Finance 2010, pp. 205-266, 2011.

[18] Y. Achdou and I. Capuzzo-Dolcetta, "Mean field games: Numerical methods," SIAM Journal on Numerical Analyss, vol. 48, no. 3, pp. 1136$1162,2010$.

[19] B. K. Øksendal, Stochastic differential equations: an introduction with applications. Springer Verlag, 2003. 